\title{
Tálamo según Galeno. Una Metáfora Controversial
}

\author{
Thalamus According to Galen. A Controversial Metaphor
}

\author{
Jorge Eduardo Duque Parra ${ }^{1,2}$; Bélgica Vásquez ${ }^{3} \&$ Mariano del Sol $^{4,5}$
}

DUQUE, P. J. E.; VÁSQUEZ, B. \& DEL SOL, M. Tálamo según Galeno. Una metáfora controversial. Int. J. Morphol., 38(3):799803, 2020.

RESUMEN: El vocabulario técnico-científico, uno de ellos la Terminologia Anatomica, tiene un legado lingüístico de idiomas clásicos en general y del latín y griego en particular. En este contexto, la metáfora ha cumplido un importante rol en la denominación de ciertas estructuras del cuerpo humano. El análisis de estas metáforas ha permitido conocer el origen etimológico de numerosos términos anatómicos derivados de esta práctica frecuente durante la historia. Este estudio tuvo como objetivo analizar y reflexionar acerca de la utilización del término tálamo y comentar la similitud formal de esta metáfora con las características neuroanatómicas. El nombre tálamo fue asignado por Claudio Galeno (130 - 200 a. C.); procede de un lenguaje común de orden material, el cual ha sido mencionado por autores clásicos, principalmente, como "cámara interna o cámara nupcial" y llevado a un lenguaje técnico-científico a través de una metáfora motivada por una disposición espacial o entendida como una expresión de imagen o similitud formal. Si Galeno utilizó esta metáfora considerando una similitud formal, el término tálamo sería equívoco, ya que no hay correspondencia estructural del término debido a que el tálamo neuroanatómico no es una cámara, sino una estructura diencefálica compacta y esferoidal u ovalada. Bajo este contexto, el término tálamo es confuso, ya que esta metáfora se condice más bien con el tercer ventrículo. Considerando lo anterior, invitamos a reflexionar sobre una propuesta basada en una característica morfológica de la estructura, en la cual se reemplace el término tálamo por neuroovoide.

PALABRAS CLAVE: Terminologia Anatomica; Tálamo; Galeno; Etimología; Metáfora.

\section{INTRODUCCIÓN}

El término tálamo se encuentra incluido desde la primera Nomina Anatomica de Basilea (BNA) de 1985 hasta la Terminologia Anatomica (2019), sufriendo durante estos 125 años diversas clasificaciones y modificaciones.

Terminologia Anatomica Internacional fue desarrollada por la Federación Internacional de Asociaciones de Anatomía (IFAA) en latín, pudiendo ser traducida al idioma vernáculo para ser utilizado por los anatomistas y neuroanatomistas que hacen uso de estos términos, teniendo en consideración que los nombres de las estructuras corporales deberían poseer un valor informativo (Vásquez \& del Sol, 2015; Duque et al., 2018).

Parte del desarrollo histórico de la Anatomía se inició con los escritos existentes en el Imperio Romano, como los de Galeno de Pérgamo (130 - 200 a. C.) de origen griego
(Jónico), considerado uno de los padres de la medicina occidental. La obra de Galeno se basó en la tradición hipocrática, a la que unió elementos del pensamiento de Platón (427 - 347 a. C.) y Aristóteles (384 - 322 a. C.), que recibió a través del estoicismo de Posidonio. Completó sus estudios en Alejandría, donde comprendió la importancia de los estudios anatómicos y fisiológicos. Tuvo contacto con las obras de anatomistas como Herófilo (335 - 280 a. C.) y Erasístrato (304 - 250 a. C.) quienes realizaron grandes aportes a la neuroanatomía (Martínez \& Decuadro-Sáenz, 2008). Además, Galeno tuvo una excelente formación que le permitió conocer en profundidad las distintas escuelas médicas del momento y añadir a todo ello sus contribuciones originales.

Galeno intentó elaborar una terminología en la que involucró lo neuroanatómico, en la cual se reconoce un componente del diencéfalo, el tálamo.

\footnotetext{
${ }^{1}$ Departamento de Ciencias Básicas. Programa de Medicina, Universidad de Manizales. Manizales, Colombia.

${ }^{2}$ Departamento de Ciencias Básicas. Programa de Medicina, Universidad de Caldas. Manizales, Colombia.

${ }^{3}$ Universidad de Tarapacá, Arica, Chile.

${ }^{4}$ Programa de Doctorado en Ciencias Morfológicas, Facultad de Medicina, Universidad de La Frontera, Temuco, Chile.

${ }^{5}$ Centro de Excelencia en Estudios Morfológicos y Quirúrgicos (CEMyQ), Universidad de La Frontera, Temuco, Chile.
} 
El nombre tálamo, fue asignado por Galeno (Hyrtl, 1880; Jones, 2003; Perea-Bartolomé \& Ladera-

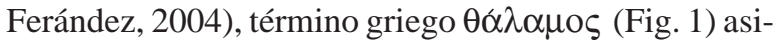
milado al latín thalamus (Morales, 2014), en relación a una estructura doble, con unos sesenta núcleos citoarquitectónicos y funcionalmente distintos, cada uno de los cuales tiene un patrón diferente de conexiones subcorticales, corticales y cerebelosos (Fama \& Sullivan, 2015) que se han definido previamente por sus propiedades quimioarquitectónicas y citoarquitectónicas; se trata de territorios discretos, funcionalmente especializados, con patrones graduados de conectividad y ordenados topográficamente (Lambert et al., 2017) que intervienen en la regulación de la información sensorial contralateral precortical cerebral, participando en la regulación de la misma (Halassa \& Acsády, 2016).

En cuanto a la denominación de las estructuras, para llegar a aquellas primeras designaciones anatómicas, los griegos, recurrieron sobre todo a procedimientos léxicos y estilísticos. El uso de los tropos, especialmente de la metáfora, es uno de los rasgos lingüísticos más destacados en aquellos primeros tiempos, de manera que la medicina antigua se sirvió con frecuencia de estas posibilidades léxicas que ofrecía la lengua griega (Moral, 2009).

En la arquitectura de la Grecia arcaica, la palabra tálamo $(\theta \alpha \lambda \alpha \mu o \varsigma)$, sirve para designar la habitación situada más al interior de una casa. Generalmente era en este espacio donde se guardaban los objetos más valiosos. Sin embargo, más tarde, en el mundo grecoromano, el término tálamo tuvo un significado que está orientado más bien a un espacio con diferentes usos (Lajo, 1990; Perea-Bartolomé \& Ladera-Fernández). Bajo este contexto, Joseph Hyrtl (1810-1894) en su Onomatología Anatómica, anota que en griego y en latín, el término tálamo tiene un significado similar: sala de estar, dormitorio (Hyrtl, 1880; Jones, 1985; Jones, 2003), cámara nupcial o aposento de los casados (Hyrtl; de Miguel \& de Morarte, 1954), en referencia al matrimonio, la consumación y la continuidad de la tribu. La palabra tálamo aparece comúnmente en la Eneida de Virgilio (70 - 19 a.n.e) y en este sentido, nos ha llegado en la poética, epithalamion. Cabe destacar que esta palabra tuvo una connotación de comportamiento sexual que se hizo muy explícito en las obras de los poetas romanos como ya se anotó con Virgilio, en el escrito Ars Amatoria de Ovidio (43 a.n.e - 17), además en las Odes de Horacio (65 a.n.e - 8), en los poemas de Propertius (50 a.n.e - 15) y en el Satiricon de Petronius (27 a.n.e - 66). En el escrito Gargantúa y Pantagruel de
Rabelais (1494-1553) se llama a la nave insignia el "Thalamége", en el viaje para pedir consejo al oráculo con respecto a la virtud de la futura esposa de Panurge, (Jones, 1985). También se usa tálamo en relación a cámara de almacén y del mismo modo, al palacio habitado por los apis egipcios; igualmente, en anatomía, fueron llamados tálamo las cámaras del corazón -Thalami cordis- (Hyrtl).

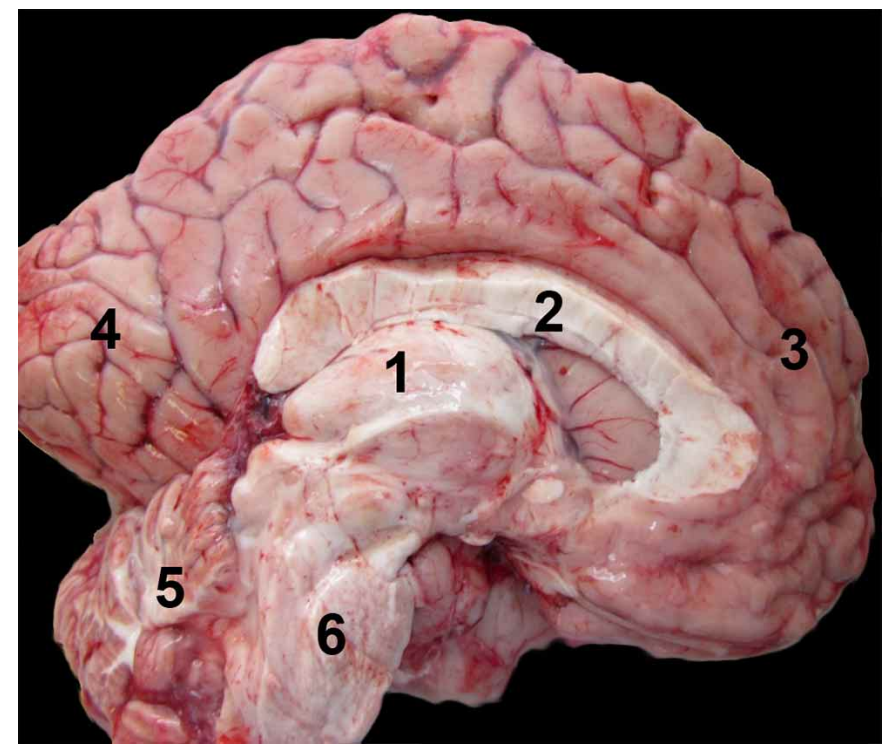

Fig.1. Corte sagital del encéfalo humano. 1. Tálamo; 2. Cuerpo calloso; 3. Lóbulo frontal del cerebro; 4. Lóbulo occipital del cerebro; 5. Cerebelo; 6. Tronco encefálico.

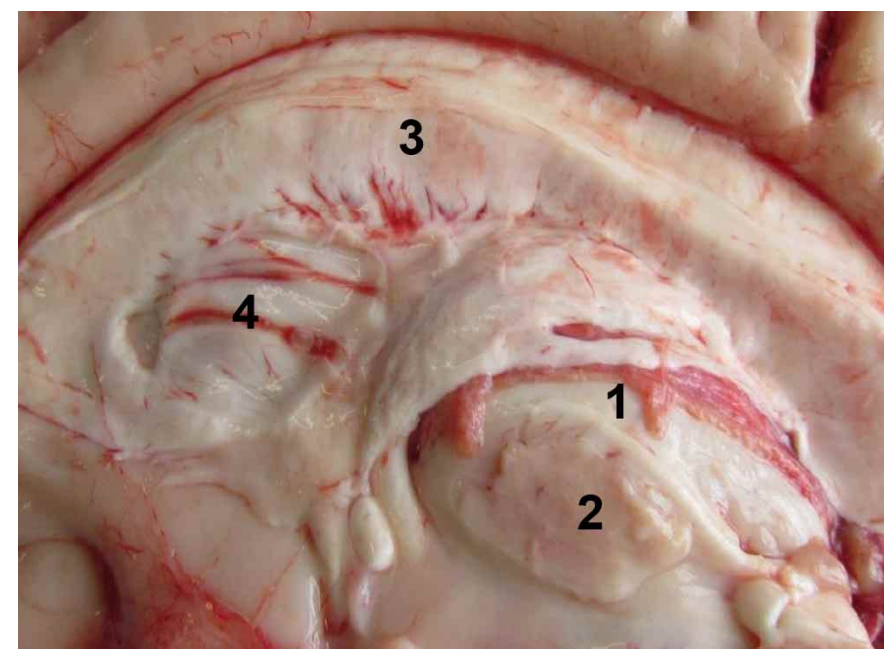

Fig. 2. Corte sagital del encéfalo humano en el que se distingue la adhesión intertalámica que separa el tercer ventrículo en dos espacios o "cámaras". 1. Tálamo; 2. Adhesión intertalámica; 3. Cuerpo calloso; 4. Septo pelúcido.

El presente trabajo pretende analizar y reflexionar sobre la metáfora tálamo para conocer el origen etimológico de este término y discutir respecto de la similitud formal de esta metáfora con las características de esta estructura neuroanatómica. 


\section{COMENTARIOS}

Recientemente, la terminología sobre el sistema nervioso central, el sistema nervioso periférico y los órganos sensoriales de Terminologia Anatomica (1998) y Terminologia Histologica (2008) se ha fusionado y actualizado ampliamente por la Programa Federativo Internacional de Terminología Anatómica (Federative International Programmee For Anatomical Terminology, FIPAT) y Grupo de trabajo sobre neuroanatomía (ten Donkelaar et al., 2018). Por otra parte, siguiendo los acuerdos y sugerencias realizadas desde la Nomina Anatomica de Basilea en 1895 y las actuales Terminologías de la FIPAT, las Sociedades Científicas, miembros de la IFAA están autorizadas a publicar traducciones de esta terminología al idiomas vernáculo de sus respectivos países.

En la BNA de 1895 solo se incluyeron tres de los núcleos del tálamo, sin embargo éstos se han ampliado en las siguientes ediciones mediante la subdivisión del núcleo lateral del tálamo y la inclusión del núcleo central del tálamo. En 1955 el Comité Internacional de Nomenclatura Anatómica publicó la Nomina Anatomica de París (PNA) donde se señaló que en dicha edición habían agregado la subdivisión del núcleo lateral del tálamo e incluido el núcleo central del tálamo. Además, en las Sectiones thalamencephali se incluyeron los núcleos talámicos.

Muchas veces, como en toda discusión terminológica, en especial la anatómica y neuroanatómica, ningún cambio o acuerdo complacerá a todos. Sin embargo, se han hecho adiciones y reordenamientos en un intento por satisfacer a la mayoría de los anatomistas y neuroanatomistas. Así en la PNA se hace mención al Thalamencephalon y al Thalamus con sus componentes pulvinar, Tuberculum anterius thalami, Taenia thalami y Stria medullaris thalami.

Chatain (1975) publicó un libro al cual denominó Terminologia Anatomica mucho años antes que la IFAA a través del International Anatomical Nomenclature Committee (IANC) adoptara este nombre como continuación de la Nomina Anatomica. Chatain señaló que la publicación incluía las recomendaciones sugeridas en el tercera edición de la Nomina Anatomica de 1966. Incluyó los tér- minos Talamencéfalo (Thalamencephalon) y Tálamo (óptico) (Thalamus), estos términos eran iguales en el nombre tradicional y nombre actual.

En la sexta edición de la Nomina Anatomica, tercera Nomina Histologica y tercera Nomina Embryologica de 1989, la cual oficialmente no alcanzó a circular, aparece mencionado el tálamo con sus componentes, tubérculo talámico anterior, adhesión intertalámica y estría medular talámica; además, indicó separadamente el tálamo dorsal con el pulvinar. También en esta Nomina Anatomica se incluye el tálamo ventral (denominado también subtálamo).

De acuerdo con la decisión en la reunión de Estambul de FIPAT (2015), y al igual que con las otras terminologías de FIPAT, se ha adoptado un nuevo formato de seis columnas. Las seis columnas comprenden el término latino oficial, sinónimo(s) latino(s) donde sea relevante, términos en inglés (Reino Unido y EE.UU.) y sinónimo(s) en inglés donde sea relevante. La columna final incluye términos relacionados, epónimos, notas direccionales y referencias a notas finales. Las notas finales se colocan en una sección separada al final de cada parte (FIPAT, 2019). Tabla I.

En el transcurso de la historia de la construcción de los términos anatómicos del cuerpo humano es posible observar el uso de la metáfora. La relevancia de esta figura retórica, consiste en que el sentido metafórico que se encuentra en ciertos términos, arroja luces sobre su etimología (Morales).

Las metáforas sirven para establecer una relación de semejanza valiéndose de un concepto o expresión que se aplica a una idea u objeto, sin describirlo de forma directa, con el fin de compararlo con otro elemento y, así, facilitar su comprensión. En otras palabras, es un recurso que representa el lenguaje humano permitiendo establecer asociaciones semánticas implícitamente relacionadas con significados que resultan conocidos (Rodríguez, 2016). De este modo, se puede afirmar que la metáfora es un tropo que consiste en presentar como idénticos términos claramente diferentes, pese a que tanto aquel que usa la metáfora como sus destinatarios son conscientes de que no existe tal identidad, sino que todo se reduce a tan solo una semejanza formal o funcional (García, 1989).

Tabla I. Término tálamo actuales en Terminologia Neuroanatomica (2017) y Terminologia Anatomica (2019).

\begin{tabular}{lcccccc}
\hline Terminologías & Término & $\begin{array}{c}\text { Sinónimo } \\
\text { latino }\end{array}$ & $\begin{array}{c}\text { Inglés } \\
\text { británico }\end{array}$ & $\begin{array}{c}\text { Inglés } \\
\text { americano }\end{array}$ & $\begin{array}{c}\text { Sinónimo } \\
\text { inglés }\end{array}$ \\
\hline $\begin{array}{l}\text { Terminologia } \\
\begin{array}{l}\text { Neuroanatomica (2017) } \\
\text { Terminologia Anatomica }\end{array}\end{array}$ & 1801 & Thalamus & & Thalamus & Thalamus & Otros \\
$(2019)$ & Thalamus & $\begin{array}{c}\text { Thalamus } \\
\text { dorsalis }\end{array}$ & Thalamus & Thalamus & $\begin{array}{c}\text { Dorsal } \\
\text { thalamus }\end{array}$ & Thalamencephalon \\
\hline
\end{tabular}


El recurso al uso de los tropos en disciplinas como la medicina, permite satisfacer las necesidades terminológicas del momento. Así, en una época en la que la medicina carecía de fundamentos científicos en el sentido más estricto del término, los "grandes maestros" hacían alarde de sus conocimientos mediante descripciones orales de estimable valor literario. Posteriormente, a medida que la medicina se fue especializando y se vio sometida a un acelerado progreso, su lenguaje perdió parte de ese léxico basado en usos culturales ancestrales, ganando en precisión, concisión y objetividad (Eurrutia, 2003).

Como el lenguaje técnico-científico de términos debe ser unívoco y preciso, no permite eludir el uso de múltiples estructuras metafóricas, sino que se debe realizar un uso discriminado en función de las necesidades del especialista (Eurrutia). El autor señala que debemos distinguir entre tropos libres (únicos, no lexicalizados), basados en el libre juego de la imaginación, frecuente en el lenguaje poético pero de escaso uso en el lenguaje técnico-científico (repetidos, lexicalizados) cuyo sentido figurado sistémico permite designar nuevas acepciones.

La mayor parte de las metáforas presentes en este lenguaje técnico-científico proceden de la lengua común. Los ámbitos conceptuales utilizados por la biomedicina como fuente metafórica con el fin de conceptualizar y denominar sus nociones son: la sociedad —orden social—, la naturaleza orden natural—, las cosas físicas creadas por el hombre orden material - y el mundo de la comunicación y la información —orden comunicativo (Díaz, 2005).

En este trabajo, la metáfora tálamo pone de manifiesto que los términos biomédicos surgen dentro de un contexto cultural determinado y que se empapan de modelos culturales procedentes de otras áreas de la cultura y ámbitos del conocimiento (Rodríguez).

En este caso, la denominación del tálamo está motivada por un metáfora del ámbito del orden material, hecha por la mano del hombre, la que puede explicarse de dos puntos de vistas, en la cual una pareciera tener más sentido que la otra, aún cuando es más escasa su evidencia en los textos clásicos.

Desde el contexto de la arquitectura de la Grecia arcaica, el tálamo es una de las partes del cerebro más importante, no sólo es una de las estructuras encefálicas más grandes, sino que además, está situado en pleno centro del encéfalo, tal y como lo refleja su nombre, que viene de la palabra griego $\theta \alpha ́ \lambda \alpha \mu$ os (cámara interna o habitación situada más al interior de una casa). Desde este punto de vista, la metáfora tálamo, realizada por Galeno, podría estar motivada por la disposición espacial: los núcleos de tejido nervio- so se sitúan en esta sección de la cabeza entendida como vivienda (Morales).

Desde otro punto de vista, Galeno podría haber considerado esta parte del sistema nervioso central como cámara. Sin embargo, el tálamo, tal como lo conocemos hoy, fue identificado como una parte del diencéfalo por los anatomistas que comenzaron a describir en detalle en el siglo XIV; el primero en describirlo fue el italiano Mondino de Luzzi (1270 1326) y las mejores ilustraciones tempranas del tálamo aparecieron en las obras de Thomas Willis (1621 - 1675). Si Galeno realmente pretendía llamar "tálamo" a la referencia para gran parte del diencéfalo, esta aseveración sería imprecisa, pues pudo haber tenido la intención de referirse a lo adyacente al tálamo, la parte que rodea al tercer ventrículo (Jones, 2003). El tálamo no es una cámara, y en este sentido Galeno podría haberlo confundido con el espacio asociado al tercer ventrículo, el cual sí hace las veces de cámara y contiene líquido cerebro espinal (Duque et al., 2018), por lo que el espacio adyacente de dicho ventrículo que rodea al tálamo que le da apariencia de una cámara, no del tejido neuroglial que de cierta forma comprime a manera de invaginación el sector central del tercer ventrículo, tanto así, que a veces se forma una adhesión intertalámica -inconstante- (Duque et al., 2014) que separa el tercer ventrículo (Fig. 2) pudiendo permitir la interpretación de que este ventrículo está constituido por dos cámaras.

En este análisis, no hay correspondencia estructural del término tálamo, lo que lo hace equívoco, y la construcción metafórica motivada por su disposición espacial no concuerda con una ubicación mental del que la interpreta. Además, los diversos núcleos talámicos descritos histológicamente, recibieron muchos de los nombres dados por Frank Alexander Nissl (1860-1919) en 1889 (Jones, 2003), los cuales han generado controversias sobre su terminología, pues en la literatura quirúrgica se usan varias, lo que complica el entendimiento para las cirugías estereotáxicas (Krack et al., 2002; Mai \& Majtanik, 2019) de trascendental importancia en neurocirugía.

Como la ciencia y, en ella la Neuroanatomía, se basa en una construcción racional para luchar contra el consenso predominante, y así, dar una especie de mejor entendimiento sobre lo que comprendemos con base en hallazgos verificados, el descubrimiento científico probado no debe admitir estos conceptos terminológicos inconsecuentes, solo por el peso de la tradición, porque Galeno lo dijo. Se deben admitir las dudas de esta idea terminológica que llevan dentro de sí una falacia, pues la invención de la palabra escrita aumentó considerablemente la exactitud y confiabilidad de los conocimientos neuroanatómicos, permitiendo corregir muchos errores, como puede ser para este caso y presentar una opción de cambio al tradicional término tálamo. 
Si algunos ya han abogado por una nomenclatura inequívoca obligatoria para la comprensión de la organización del tálamo (Mai \& Majtanik), por qué no también abogar por una, para que involucre su nombre general de tálamo?, así, puesto que el tálamo presenta forma esferoidea (Mai \& Majtanik) u ovalada (Perea-Bartolomé \& Ladera-Fernández) u ovoide (Párraga, 2012), proponemos en correspondencia al latín, el término neuroovoloide que implica una estructura oval (ovo asociado con el latín ovum, el sufijo oide relacionado con forma de, y neuro por ser un constituyente del sistema nervioso) por la forma geométrica de paraboloide que presenta. Esto implicaría a su vez modificar los nombres de estructuras diencefálicas asociadas como el hipotálamo, el metatálamo, el epitálamo y el subtálamo, así como elementos relacionados como proyecciones tálamo corticales, cortico talámicas, entre otras.

Nos atrevemos a pensar con este ligero análisis, que por generar un choque conceptual fuerte que implica cambios, los neuroanatomistas objetarán realizar un cambio, manteniendo la tradición equívoca terminológica actual, pero quizá mentes más agudas y objetivas del futuro al estudiar estas líneas, consideren esta real objeción a la tradición sin razonamiento, que suele mantenerse en el campo terminológico anatómico: esperamos que no demore mucho.

DUQUE, P. J. E.; VÁSQUEZ, B. \& DEL SOL, M. Thalamus according to Galen. A controversial metaphor. Int. J. Morphol., 38(3):709-803, 2020.

SUMMARY: The technical-scientific vocabulary, one of them the Anatomical Terminology, has a linguistic legacy of classical languages in general and of Latin and Greek in particular. In this context, the metaphor has played an important role in the naming of certain structures of the human body. The analysis of these metaphors has allowed us to know the etymological origin of numerous anatomical terms derived from this frequent practice throughout history. The purpose of this study was to analyze and reflect on the use of the term thalamus and to comment on the formal similarity of this metaphor with the neuroanatomical characteristics. The name thalamus was assigned by Claudio Galeno (130-200 BC); It comes from a common language of material order, which has been mentioned by classical authors, mainly, as "internal chamber or bridal chamber" and brought to a technicalscientific language through a metaphor motivated by a spatial arrangement or understood as a image expression or formal similarity. If Galen used this metaphor considering a formal similarity, the term thalamus would be misleading, since there is no structural correspondence to the term because the neuroanatomical thalamus is not a chamber, but a compact, spheroidal or oval diencephalic structure. In this context, the term thalamus is confusing, since this metaphor is more consistent with the third ventricle. Considering the above, we invite you to reflect on a proposal based on a morphological characteristic of the structure, in which the term thalamus is replaced by neuroovoid.

KEY WORDS: Anatomical Terminology; Thalamus; Galen; Etymology; Metaphor.

\section{REFERENCIAS BIBLIOGRÁFICAS}

Chatain, I. Terminologia Anatomica. Fernando Aldape Barrera, México, 1975. de Miguel, R.; de Morarte, M. Diccionario Latino-Español Etimológico. 27 ed. Madrid, Librería general Victoriano de Suarez; 1954.

Díaz, R. J. A. Terminología médica, cultura e ideología. Quaderns de filología Estudis lingüistics, 10:31-51, 2005.

Duque, P. J. E.; Marín, A. F. \& Duque, V. I. L. Interthalamic adhesion: An approach from basic science to implement clinic neuroimaging. 3 ed. European Conference on Clinical Neuroimaging, 2014.

Duque, P. J. E.; Barco, R. J. \& Duque, Q. M. La eponimia, ese difícil obstáculo que aún no supera la Terminología Anatómica Internacional. Int. J. Morphol., 36(4):1206-9, 2018.

Eurrutia, C. M. Formación indirecta de la terminología técnico-científica: alusiones, imágenes y metáforas. In Ignacio Iñarrea Las Heras y María Jesús Salinero Cascante (coords.): El texto como encrucijada: estudios franceses y francófonos La Rioja, Universidad de La Rioja, vol. 2, 2003. pp. 367-80.

Fama, R. \& Sullivan, E. V. Thalamic structures and associated cognitive functions Relations with age and aging. Neurosci. Biobehav. Rev., 54:29-37, 2015.

Federative Committee on Anatomical Terminology (FCAT). Terminologia Anatomica: International Anatomical Terminology. Stuttgart, Georg Thieme Verlag, 1998.

FIPAT. Federative International Programme for Anatomical Terminology. Terminologia Anatomica. $2^{\text {nd }}$ ed. fipat.library.dal.ca, 2017.

García, Y. V. Teoría y Práctica de la Traducción. Madrid, Gredos, 1989.

Halassa, M. M. \& Acsády. L. Thalamic inhibition: Diverse sources, diverse scales. Trends Neurosci., 39(10):680-93, 2016.

Hyrtl, J. Onomatologia anatomica. Geschichte und kritik der anatomischen sprache der gegenwart. Wien, Wilhelm Braumüller. K.K. Hof- und Universitätsbuchhändler, 1880.

International Anatomical Nomenclature Committee. Nomina Anatomica. Sixth ed Chuchill Livingstone, Singapore, 1989.

Jones, E. G. The History of the Thalamus. In. Jones, E. G. The Thalamus. New York, Plenum Press, 1985.

Jones, E. G. History of Neuroscience: The Thalamus. IBRO History of Neuroscience, 2003. Disponible en: http://ibro.org/wp-content/uploads/2018/07/TheThalamus.pdf.

Krack, P.; Dostrovsky, J.; Ilinsky, I.; Kultas-Ilinsky, K.; Lenz, F.; Lozano, A. \& Vitek, J. Surgery of the motor thalamus: problems with the present nomenclatures. Mov. Disord., 17 Suppl 3:S2-8, 2002.

Lajo, P. R. Léxico de Arte. Madrid, Akal, 1990. p. 194.

Lambert, C.; Simon, H.; Colman, J. \& Barrick, T. R. Defining thalamic nuclei and topographic connectivity gradients in vivo. Neuroimage, 158:466-79, 2017.

Mai, J. K. \& Majtanik, M. Toward a common terminology for the thalamus. Front. Neuroanat., 12:114, 2019.

Moral, L. M. Algunas aportaciones sobre el vocabulario médico de procedencia griega. Seminario Médico, 61(2):37-60, 2009.

Morales, H. R. Metáforas motivadas por la similitud formal en la terminología médica. Rev. Káñina, 38:113-126, 2014.

Martínez, F. \& Decuadro-Sáenz, G. Claudio Galeno y los ventrículos cerebrales. Parte I, los antecedentes. Neurocirugía, 19(1):58-65, 2008.

Párraga, R. G. Microsurgical anatomy of the lateral ventricles in three-dimensional images. Rev. Investing. Inf. Salud, 7(18):11-27, 2012.

Perea-Bartolomé, M. V. \& Ladera-Fernández, V. El tálamo: aspectos neurofuncionales. Rev. Neurol., 38(7):687-93, 2004.

Rodríguez, V. M. Estudio español-francés de la metaforización biomédica en las guías de práctica clínica en el ámbito de las enfermedades raras.Panace@, 17(44):144-9, 2016.

ten Donkelaar, H. J.; Kachlík, D.\& Tubbs, R. S. An illustrated Terminologia Neuroanatomica. Gewerbestrasse, Cham, Springer, 2018.

Vásquez, B. \& del Sol, M. Terminologia Anatomica and Terminologia Histologica. A meeting point between morphologists. Int. J. Morphol., 34(4):1585-90, 2015.

Dirección para correspondencia:

Dra. Bélgica Vásquez

Universidad de Tarapacá

Arica - CHILE

Recibido : 04-06-2019

Aceptado: 03-01-2020 